\title{
The $t$-improper chromatic number of random graphs
}

\author{
Ross J. Kang * Colin McDiarmid ${ }^{\dagger}$
}

September 2, 2018

\begin{abstract}
We consider the $t$-improper chromatic number of the Erdös-Rényi random graph $G_{n, p}$. The t-improper chromatic number $\chi^{t}(G)$ is the smallest number of colours needed in a colouring of the vertices in which each colour class induces a subgraph of maximum degree at most $t$. If $t=0$, then this is the usual notion of proper colouring. When the edge probability $p$ is constant, we provide a detailed description of the asymptotic behaviour of $\chi^{t}\left(G_{n, p}\right)$ over the range of choices for the growth of $t=t(n)$.
\end{abstract}

\section{Introduction}

We consider the $t$-improper chromatic number of the Erdős-Rényi random graph $G_{n, p}$. As usual, $G_{n, p}$ denotes a random graph with vertex set $[n]=\{1, \ldots, n\}$ in which the edges are included independently at random with probability $p$. The $t$-dependence number $\alpha^{t}(G)$ of a graph $G$ is the maximum size of a $t$-dependent set - a vertex subset which induces a subgraph of maximum degree at most $t$. The $t$-improper chromatic number $\chi^{t}(G)$ is the smallest number of colours needed in a t-improper colouring - a colouring of the vertices in which colour classes are $t$-dependent sets. Note that $\chi^{t}(G) \geq|V(G)| / \alpha^{t}(G)$ for any graph $G$ and any integer $t$.

The $t$-improper chromatic number was introduced about two decades ago independently by Andrews and Jacobson [1], Harary and Fraughnaugh (née Jones) [11, 12], and Cowen et al. [6]. In the first paper, the authors considered various general lower bounds for the $t$ improper chromatic number; in the second, the authors studied $\chi^{t}$ as part of the larger setting of generalised chromatic numbers; in the third, the authors established best upper bounds on $\chi^{t}$ for planar graphs to generalise the Four Colour Theorem. Several papers on the topic have since appeared; for instance, two papers, by Eaton and Hull [8] and Škrekovski [22], extend the program of Cowen et al. to a list colouring variant $\operatorname{ch}^{t}$ of $\chi^{t}$ and both pose the question: is $\operatorname{ch}^{1}(G) \leq 4$ for every planar graph $G$ ?

Clearly, when $t=0$, we are simply considering the ordinary notion of the chromatic number of random graphs, and this topic is well studied. Fix $0<p<1$ and let $b=$ $1 /(1-p)$. In 1975, Grimmett and McDiarmid [10] conjectured that $\chi\left(G_{n, p}\right) \sim n /\left(2 \log _{b} n\right)$ asymptotically almost surely (a.a.s.). This remained a major open problem in random graph theory for over a decade, until Bollobás [2] and Matula and Kučera [19] used martingale

\footnotetext{
${ }^{*}$ School of Computer Science, McGill University, Montréal, Québec, H2A 2A7, Canada. Part of this work was completed while this author was a doctoral student at the University of Oxford. He was partially supported by NSERC (Canada) and the Commonwealth Scholarships Commission (UK).

${ }^{\dagger}$ Department of Statistics, University of Oxford, 1 South Parks Road, Oxford OX1 3TG, United Kingdom.
} 
techniques to establish the conjecture. Łuczak [18] extended the result to sparse random graphs. For further background into the colouring of random graphs, consult $[13,3]$. The main objective of this paper is to extend this study to $t$-improper colouring.

Before we describe our main results, let us make some basic observations about the behaviour of the $t$-improper chromatic number. Let $G$ be a graph and $t$ a non-negative integer. Since a $t$-dependent set is $(t+1)$-dependent, it follows that $\chi^{t}(G) \geq \chi^{t+1}(G)$. Also, since each colour class of a $t$-improper colouring is $t$-dependent and so can be properly coloured with at most $t+1$ colours, it follows that $\chi^{t}(G) \geq \chi(G) /(t+1)$. Furthermore, it is straightforward to derive from a decomposition theorem of Lovász [17] (cf. [5]) that $\chi^{t}(G) \leq\lceil(\Delta(G)+1) /(t+1)]$ where $\Delta(G)$ denotes the maximum degree of $G$. As a consequence of these last three observations, we obtain the following range of values for $\chi^{t}(G)$.

Proposition 1. For any graph $G$ and non-negative integer $t$,

$$
\frac{\chi(G)}{t+1} \leq \chi^{t}(G) \leq \min \left\{\left\lceil\frac{\Delta(G)+1}{t+1}\right\rceil, \chi(G)\right\} .
$$

In this paper, our main focus is on dense random graphs - i.e. the case in which the edge probability $p$ is a fixed constant between 0 and 1 . Recall that $\Delta\left(G_{n, p}\right) \sim n p$ a.a.s. in this case. We show that $\chi^{t}\left(G_{n, p}\right)$ is likely to be close to the upper end of the range in Proposition 1, as long as $t(n)=o(\ln n)$ or $t(n)=\omega(\ln n)$. We also give a precise description of the behaviour of $\chi^{t}\left(G_{n, p}\right)$ for the intermediate case $t(n)=\Theta(\ln n)$. Here is our main theorem.

Theorem 2. Fix $0<p<1$ and let $b=1 /(1-p)$. There exists a function $\kappa_{p}=\kappa_{p}(\tau)$ that is continuous and strictly increasing for $\tau \in[0, \infty)$, with $\kappa_{p}(0)=2 / \ln b$ and $\kappa_{p}(\tau) \sim \tau / p$ as $\tau \rightarrow \infty$ such that the following holds: a.a.s.

$$
\chi^{t}\left(G_{n, p}\right) \sim \frac{n}{\kappa_{p}(t / \ln n) \ln n}
$$

if $t(n)=o(n)$. Furthermore, if $t(n) \sim n p / x$, where $x>0$ is fixed and not integral, then $\chi^{t}\left(G_{n, p}\right)=\lceil x\rceil$ a.a.s.

In Sections 2 and 3, we use large deviations techniques and a second moment calculation to develop a fairly precise description of the $t$-dependence number of $G_{n, p}$. By these computations, we identify the function $\kappa_{p}$. A proof of the main theorem is given in Section 4.

For sparse random graphs - i.e. when $p(n)=o(1)$ — we will give just one result. For further results on the $t$-improper chromatic number in this regime, see Section 4.3 of [14].

Theorem 3. Suppose $0<p(n)<1, p(n)=o(1)$ and $\varepsilon>0$. Set $d(n)=n p(n)$. There exist constants $d_{0}$ and $\tau>0$ such that, if $d(n) \geq d_{0}$ and $t(n) \leq \tau \ln d$, then $(1-\varepsilon) d /(2 \ln d) \leq$ $\chi^{t}\left(G_{n, p}\right) \leq(1+\varepsilon) d /(2 \ln d)$ a.a.s.

The upper bound here follows immediately from the upper bound of Euczak [18] on the chromatic number of sparse random graphs. A corollary is that, if $d(n) \rightarrow \infty$ and $t(n)=$ $o(\ln d)$ as $n \rightarrow \infty$, then $\chi^{t}\left(G_{n, p}\right) \sim d /(2 \ln d)$ a.a.s. We shall prove Theorem 3 in Section 5.

For fixed $t$, the property of a set being $t$-dependent is an hereditary property so that the results of Scheinerman [21] and Bollobás and Thomason [4] apply — but in our work it is important that $t$ is allowed to vary. 


\section{The expected number of $t$-dependent $k$-sets}

In this section, we use large deviations results to describe the behaviour of the expected number of $t$-dependent $k$-sets. This estimation provides us with an immediate lower bound for $\chi^{t}\left(G_{n, p}\right)$. For background into large deviations, consult [7]; we borrow some notation from this reference. Given $0<p<1$, we let $q=1-p$ throughout. Also, let

$$
\Lambda^{*}(x)= \begin{cases}x \ln \frac{x}{p}+(1-x) \ln \frac{1-x}{q} & \text { for } x \in[0,1] \\ \infty & \text { otherwise }\end{cases}
$$

(where $\Lambda^{*}(0)=\ln (1 / q)$ and $\Lambda^{*}(1)=\ln (1 / p)$ ). This is the Fenchel-Legendre transform of the logarithmic moment generating function associated with the Bernoulli distribution with probability $p$ (cf. Exercise 2.2.23(b) of [7]). Some easy calculus checks that $\Lambda^{*}(x)$ has a global minimum of 0 at $x=p$, is strictly decreasing on $[0, p)$ and strictly increasing on $(p, 1]$. We note the following large deviations result for the binomial distribution.

Lemma 4. There is a constant $\delta>0$ such that the following holds. Let $0<p<1$, let $n$ be a positive integer, and let $X \in \operatorname{Bin}(n, p)$. Then for each positive integer $k \leq n p$,

$$
\delta \cdot \max \left\{k^{-1 / 2},(n-k)^{-1 / 2}\right\} \cdot \exp \left(-n \Lambda^{*}(k / n)\right) \leq \mathbb{P}(X \leq k) \leq \exp \left(-n \Lambda^{*}(k / n)\right) .
$$

Also, of course $\mathbb{P}(X=0)=q^{n}=\exp \left(-n \Lambda^{*}(0)\right)$. Furthermore, the monotonicity of $\Lambda^{*}(x)$ shows that the right inequality holds also for non-integral $k$. For convenience, we give a proof of this lemma in the appendix to this paper. For related very general results, see for example the monograph of Dembo and Zeitouni [7]. Lemma 4 immediately yields the following estimate for the number of $t$-dependent $k$-sets. For a graph $G$, we let $\overline{\operatorname{deg}}(G) \operatorname{denote}$ the average degree of $G$.

Lemma 5. Suppose $0<p=p(n)<1$ and suppose the positive integers $t=t(n)$ and $k=k(n)$ satisfy that $t \leq p(k-1)$.

(i) $\mathbb{P}\left(\overline{\operatorname{deg}}\left(G_{k, p}\right) \leq t\right) \leq \exp \left(-\left(\begin{array}{l}k \\ 2\end{array}\right) \Lambda^{*}\left(\frac{t}{k-1}\right)\right)$; and

(ii) $\mathbb{P}\left(\overline{\operatorname{deg}}\left(G_{k, p}\right) \leq t\right) \geq \exp \left(-\left(\begin{array}{l}k \\ 2\end{array}\right) \Lambda^{*}\left(\frac{t}{k-1}\right)-\ln k+O(1)\right)$.

Since a $t$-dependent $k$-set has average degree at most $t$, Lemma 5(i) implies an upper bound on the expected $t$-dependence number of $G_{n, p}$. In particular, it shows that, if

$$
\left(\begin{array}{l}
n \\
k
\end{array}\right) \exp \left(-\left(\begin{array}{l}
k \\
2
\end{array}\right) \Lambda^{*}\left(\frac{t}{k-1}\right)\right)=o(1)
$$

then $\alpha^{t}\left(G_{n, p}\right) \leq k$ a.a.s. We define the function $\kappa_{p}(\tau)$ of Theorem 2 based on the range of $k$ (given $t$ ) for which the above condition holds. Note first the following lemma, the straightforward proof of which is omitted.

Lemma 6. Fix $0<p<1$. For any $\tau \geq 0$, there is a unique $\kappa_{p}(\tau)>\tau / p$ such that

$$
\frac{\kappa}{2} \Lambda^{*}\left(\frac{\tau}{\kappa}\right)\left\{\begin{array}{ll}
<1 & \text { if } \tau / p<\kappa<\kappa_{p}(\tau) \\
=1 & \text { if } \kappa=\kappa_{p}(\tau) \\
>1 & \text { if } \kappa>\kappa_{p}(\tau)
\end{array} .\right.
$$


The function $\kappa_{p}(\tau)$ for $\tau \in[0, \infty)$ is continuous and strictly increasing, with $\kappa_{p}(0)=2 / \ln (1 / q)$ and $\kappa_{p}(\tau) \sim \tau / p$ as $\tau \rightarrow \infty$.

Suppose $t(n) / \ln n \rightarrow \tau$ for some $\tau \geq 0$. The main result of this section is to show that, if $\tau / p<\kappa<\kappa_{p}(\tau)$, then the expected number of $t$-dependent $(\kappa \ln n)$-sets goes to infinity and, if $\kappa>\kappa_{p}(\tau)$, then it goes to zero. More precisely, we have the following.

Theorem 7. Fix $0<p<1$. Fix $\tau, \kappa \geq 0$ with $\kappa>\tau / p$ and suppose $t(n) / \ln n \rightarrow \tau$ as $n \rightarrow \infty$ and $k(n) \sim \kappa \ln n$. Let $\mathcal{S}_{n, t, k}$ be the collection of $t$-dependent $k$-sets in $G_{n, p}$. Then

$$
\mathbb{E}\left(\left|\mathcal{S}_{n, t, k}\right|\right)=\exp \left(k \ln n\left(1-\frac{\kappa}{2} \Lambda^{*}\left(\frac{\tau}{\kappa}\right)+o(1)\right)\right) .
$$

Before continuing with the proof of this theorem, we mention that there is a similar statement for the expected number of $t$-improper $\lceil n / k\rceil$-colourings of $G_{n, p}$. That is, under the same conditions as Theorem 7 , if $\mathcal{C}_{n, t, k}$ is the collection of $t$-improper $\lceil n / k\rceil$-colourings of $G_{n, p}$, then

$$
\mathbb{E}\left(\left|\mathcal{C}_{n, t, k}\right|\right)=\exp \left(n \ln n\left(1-\frac{\kappa}{2} \Lambda^{*}\left(\frac{\tau}{\kappa}\right)+o(1)\right)\right) .
$$

A proof of this statement can be found in Section 4.2 of [14]. Observe that this generalises a result of Grimmett and McDiarmid [10] concerning the expected number of proper $j$-colourings of $G_{n, p}$.

Proof of Theorem 7. Clearly, since $\kappa>\tau / p$, it follows that $t(n) \leq p(k(n)-1)$ for large enough $n$. Thus, since $(n / k)^{k} \leq\left(\begin{array}{l}n \\ k\end{array}\right) \leq(e n / k)^{k}$, it follows from Lemma $5(\mathrm{i})$ and the continuity of $\Lambda^{*}$ that

$$
\mathbb{E}\left(\left|\mathcal{S}_{n, t, k}\right|\right) \leq\left(\begin{array}{l}
n \\
k
\end{array}\right) \exp \left(-\left(\begin{array}{l}
k \\
2
\end{array}\right) \Lambda^{*}\left(\frac{t}{k-1}\right)\right)=\exp \left(k \ln n\left(1-\frac{\kappa}{2} \Lambda^{*}\left(\frac{\tau}{\kappa}\right)+o(1)\right)\right)
$$

and so we just need to show the reverse inequality.

Our approach will be to bound the probability that a $k$-set is $t$-dependent with an appropriately chosen conditional probability. First, we will give an estimate for the conditional probability

$$
P_{n, \varepsilon}=\mathbb{P}\left(\Delta\left(G_{k, p}\right)>t \mid \overline{\operatorname{deg}}\left(G_{k, p}\right) \leq(1-\varepsilon) t\right)
$$

for $0<\varepsilon<1$. Note that, if we condition on a fixed number $m \in\left\{0, \ldots,\left(\begin{array}{l}k \\ 2\end{array}\right)\right\}$ of edges in $G_{k, p}$, this is essentially the uniform random graph model $G_{k, m}$ (where we choose among all $\left(\begin{array}{c}k \\ 2 \\ m\end{array}\right)$ possible subgraphs with $m$ edges $)$. Thus, $\mathbb{P}\left(\Delta\left(G_{k, p}\right)>t|| E\left(G_{k, p}\right) \mid=m\right)=\mathbb{P}\left(\Delta\left(G_{k, m}\right)>\right.$ $t)$. Also, it is clear by a coupling argument that, if there are more edges, then it is more likely that the maximum degree will be higher, i.e. $\mathbb{P}\left(\Delta\left(G_{k, m-1}\right)>t\right) \leq \mathbb{P}\left(\Delta\left(G_{k, m}\right)>t\right)$. Now let $\hat{m}=\lfloor(1-\varepsilon) k t / 2\rfloor$. It follows that

$$
\begin{aligned}
P_{n, \varepsilon} & =\mathbb{P}\left(\Delta\left(G_{k, p}\right)>t|| E\left(G_{k, p}\right) \mid \leq \hat{m}\right) \\
& \leq \mathbb{P}\left(\Delta\left(G_{k, \hat{m}}\right)>t\right) \\
& \leq k \mathbb{P}\left(\operatorname{deg}(v)>t \text { in } G_{k, \hat{m}}\right) .
\end{aligned}
$$


The degree of a vertex in $G_{k, \hat{m}}$ has a hypergeometric distribution with parameters $\left(\begin{array}{l}k \\ 2\end{array}\right), k-1$ and $\hat{m}$ with expected value $\lambda=(k-1) \hat{m} /\left(\begin{array}{c}k \\ 2\end{array}\right) \leq(1-\varepsilon) t$; and thus, by a Chernoff-Hoeffding inequality (cf. Theorem 2.10 and Inequality (2.5) of [13]),

$$
P_{n, \varepsilon} \leq k \exp \left(-\frac{\varepsilon^{2} t^{2}}{2 t(1-2 \varepsilon / 3)}\right) \leq k \exp \left(-\frac{\varepsilon^{2}}{2} t\right)
$$

If we choose $\varepsilon=\varepsilon_{n}$ approaching zero slowly enough, say, $\varepsilon_{n}=(\ln n)^{-1 / 3}$, then this conditional probability is $o(1)$. Then, furthermore, using Lemma 5(ii),

$$
\begin{aligned}
\mathbb{P}\left(\Delta\left(G_{k, p}\right) \leq t\right) & \geq \mathbb{P}\left(\Delta\left(G_{k, p}\right) \leq t \mid \overline{\operatorname{deg}}\left(G_{k, p}\right) \leq\left(1-\varepsilon_{n}\right) t\right) \cdot \mathbb{P}\left(\overline{\operatorname{deg}}\left(G_{k, p}\right) \leq\left(1-\varepsilon_{n}\right) t\right) \\
& =\left(1-P_{n, \varepsilon_{n}}\right) \mathbb{P}\left(\overline{\operatorname{deg}}\left(G_{k, p}\right) \leq\left(1-\varepsilon_{n}\right) t\right) \\
& \geq(1-o(1)) \exp \left(-\left(\begin{array}{l}
k \\
2
\end{array}\right)\left(\Lambda^{*}\left(\frac{\tau}{\kappa}\right)+o(1)\right)\right)\left[\text { since } \varepsilon_{n} \rightarrow 0 \text { slowly enough }\right] \\
& =\exp \left(-\left(\begin{array}{c}
k \\
2
\end{array}\right)\left(\Lambda^{*}\left(\frac{\tau}{\kappa}\right)+o(1)\right)\right)
\end{aligned}
$$

and the expected number of $t$-dependent $k$-sets satisfies

$$
\begin{aligned}
\mathbb{E}\left(\left|\mathcal{S}_{n, t, k}\right|\right) & \geq\left(\begin{array}{l}
n \\
k
\end{array}\right) \exp \left(-\left(\begin{array}{l}
k \\
2
\end{array}\right)\left(\Lambda^{*}\left(\frac{\tau}{\kappa}\right)+o(1)\right)\right) \\
& =\exp \left(k \ln n\left(1-\frac{\kappa}{2} \Lambda^{*}\left(\frac{\tau}{\kappa}\right)+o(1)\right)\right)
\end{aligned}
$$

as required.

\section{A second moment calculation}

In this section, we perform a second moment calculation which yields both a lower bound on the $t$-dependence number, as well as an upper bound on the $t$-improper chromatic number, for the case when $t(n)$ is of order $\ln n$. We remark that the following lemma was posed as a conjecture in an earlier version of this work [15].

Lemma 8. Fix $0<p<1$. Suppose $t(n) / \ln n \rightarrow \tau$ as $n \rightarrow \infty$ for some fixed $\tau \geq 0$. If $\kappa_{p}(\tau)$ is as in Lemma 6 , then $\chi^{t}\left(G_{n, p}\right) \sim n /\left(\kappa_{p}(\tau) \ln n\right)$ a.a.s.

In a parallel work together with N. Fountoulakis [9], we have obtained a very precise description of both the $t$-dependence and the $t$-improper chromatic numbers of $G_{n, p}$ in the case when $t$ and $p$ are both fixed constants. We shall borrow some techniques from Section 4 of that work to show the following lemma.

Lemma 9. Fix $0<p<1$. Suppose $t(n) / \ln n \rightarrow \tau$ as $n \rightarrow \infty$ for some fixed $\tau \geq 0$. If $\kappa_{p}(\tau)$ is as in Lemma 6 and $k / \ln n \rightarrow \kappa_{p}(\tau)-\varepsilon$ as $n \rightarrow \infty$ for some fixed $\varepsilon>0$, then $\mathbb{P}\left(\alpha^{t}\left(G_{n, p}\right)<k\right) \leq \exp \left(-\Omega\left(n^{2} /(\ln n)^{5}\right)\right)$ a.a.s.

Establishing a similar lemma for the ordinary chromatic number was key in [2] to pinning down the asymptotic behaviour of the chromatic number of $G_{n, p}$. Before proceeding with the proof of this lemma, let us see how it implies Lemma 8. 
Proof of Lemma 8. For the lower bound, let $\kappa>\kappa_{p}(\tau)$. If we let $\delta=(\kappa / 2) \Lambda^{*}(\tau / \kappa)-1$, then $\delta>0$ by Lemma 6 . But now, setting $k=\lceil\kappa \ln n\rceil$, we have from Theorem 7 that

$$
\mathbb{P}\left(\chi^{t}\left(G_{n, p}\right) \leq \frac{n}{\kappa \ln n}\right) \leq \mathbb{P}\left(\alpha^{t}\left(G_{n, p}\right) \geq k\right) \leq \mathbb{E}\left(\left|\mathcal{S}_{n, t, k}\right|\right)=\exp (-(\delta+o(1)) k \ln n)
$$

(where $\mathcal{S}_{n, t, k}$ is the collection of $t$-dependent $k$-sets in $\left.G_{n, p}\right)$; thus, $\chi^{t}\left(G_{n, p}\right) \geq n /(\kappa \ln n)$ a.a.s.

Now for the upper bound, suppose $k=\lceil\kappa \ln n\rceil$, where $\kappa=\kappa_{p}(\tau)-\varepsilon / 2>0$ for some fixed $\varepsilon>0$. Let $\mathcal{A}_{n}$ denote the set of graphs $G$ on $[n]$ such that $\alpha^{t}(G[S]) \geq k$ for all $S \subseteq[n]$ with $|S| \geq n /(\ln n)^{2}$. Then, by Lemma 9 ,

$$
\mathbb{P}\left(G_{n, p} \notin \mathcal{A}_{n}\right) \leq 2^{n} \mathbb{P}\left(\alpha^{t}\left(G_{\left\lceil n /(\ln n)^{2}\right\rceil, p}\right)<k\right) \leq \exp \left(O(n)-\Omega\left(n^{2} /(\ln n)^{9}\right)\right) \rightarrow 0
$$

as $n \rightarrow \infty$. Therefore, $G_{n, p} \in \mathcal{A}_{n}$ a.a.s.

But for a graph $G$ in $\mathcal{A}_{n}$ the following procedure will yield a colouring as desired. Let $S^{\prime}=[n]$. While $\left|S^{\prime}\right| \geq n /(\ln n)^{2}$, form a colour class from an arbitrary $t$-dependent $k$-subset $T$ of $S^{\prime}$ and let $S^{\prime}=S^{\prime} \backslash T$. At the end of these iterations, $\left|S^{\prime}\right|<n /(\ln n)^{2}$ and we may just assign each vertex of $S^{\prime}$ to its own colour class. The resulting partition is a $t$-improper colouring of $G_{n, p}$ and the total number of colours used is less than $n /\left(\left(\kappa_{p}(\tau)-\varepsilon / 2\right) \ln n\right)+n /(\ln n)^{2} \leq$ $n /\left(\left(\kappa_{p}(\tau)-\varepsilon\right) \ln n\right)$ for large enough $n$.

For the proof of Lemma 9, it is convenient to introduce one lemma, which is proved in the appendix.

Lemma 10. Let $n_{1}$ and $n_{2}$ be positive integers, let $0<p<1$, and let $X$ and $Y$ be independent random variables with $X \in \operatorname{Bin}\left(n_{1}, p\right)$ and $Y / 2 \in \operatorname{Bin}\left(n_{2}, p\right)$. Note that $\mathbb{E}(X+Y)=\left(n_{1}+\right.$ $\left.2 n_{2}\right) p$. Then for $0 \leq x \leq p$

$$
\mathbb{P}\left(X+Y \leq\left(n_{1}+2 n_{2}\right) x\right) \leq \exp \left(-\frac{1}{2}\left(n_{1}+2 n_{2}\right) \Lambda^{*}(x)\right) .
$$

For comparison, note that, if instead of $Y / 2 \in \operatorname{Bin}\left(n_{2}, p\right)$ we had $Y \in \operatorname{Bin}\left(2 n_{2}, p\right)$, then $X+Y \in \operatorname{Bin}\left(n_{1}+2 n_{2}, p\right)$ and so $\mathbb{P}\left(X+Y \leq\left(n_{1}+2 n_{2}\right) x\right) \leq \exp \left(-\left(n_{1}+2 n_{2}\right) \Lambda^{*}(x)\right)$ by Lemma 4.

Proof of Lemma 9. We may assume without loss of generality that $0<\varepsilon<\kappa_{p}(\tau)-\tau / p$. Let $\mathcal{S}_{n, t, k}$ be the collection of $t$-dependent $k$-sets in $G_{n, p}$. Let $\kappa=\kappa_{p}(\tau)-\varepsilon$. Notice that $1-(\kappa / 2) \Lambda^{*}(\tau / \kappa)=\delta$ for some fixed $\delta>0$ by Lemma 6 ; thus, by Theorem 7 ,

$$
\mathbb{E}\left(\left|\mathcal{S}_{n, t, k}\right|\right)=\exp \left((\delta \kappa+o(1))(\ln n)^{2}\right) .
$$

We use Janson's Inequality (Theorem 2.18(ii) in [13]):

$$
\mathbb{P}\left(\alpha^{t}\left(G_{n, p}\right)<k\right)=\mathbb{P}\left(\left|\mathcal{S}_{n, t, k}\right|=0\right) \leq \exp \left(-\frac{\left(\mathbb{E}\left(\left|\mathcal{S}_{n, t, k}\right|\right)\right)^{2}}{\mathbb{E}\left(\left|\mathcal{S}_{n, t, k}\right|\right)+\Delta}\right)
$$

where

$$
\Delta=\sum_{A, B \subseteq[n], 1<|A \cap B|<k} \mathbb{P}\left(A, B \in \mathcal{S}_{n, t, k}\right) .
$$


Let $p(k, \ell)$ be the probability that two $k$-subsets of $[n]$ that overlap on exactly $\ell$ vertices are both in $\mathcal{S}_{n, t, k}$. We write

$$
\Delta=\sum_{\ell=2}^{\lfloor\lambda \ln n\rfloor}\left(\begin{array}{l}
n \\
k
\end{array}\right)\left(\begin{array}{l}
k \\
\ell
\end{array}\right)\left(\begin{array}{l}
n-k \\
k-\ell
\end{array}\right) p(k, \ell)+\sum_{\ell=\lfloor\lambda \ln n\rfloor+1}^{k-1}\left(\begin{array}{l}
n \\
k
\end{array}\right)\left(\begin{array}{l}
k \\
\ell
\end{array}\right)\left(\begin{array}{l}
n-k \\
k-\ell
\end{array}\right) p(k, \ell):=\Delta_{1}+\Delta_{2} .
$$

for some fixed $\lambda$ to be specified later.

We first bound $\Delta_{1}$. Let $A$ and $B$ be two $k$-subsets of $[n]$ that overlap on exactly $\ell$ vertices, i.e. $|A \cap B|=\ell$. Then $p(k, \ell)=\mathbb{P}\left(A, B \in \mathcal{S}_{n, t, k}\right)=\mathbb{P}\left(A \in \mathcal{S}_{n, t, k} \mid B \in \mathcal{S}_{n, t, k}\right) \mathbb{P}\left(B \in \mathcal{S}_{n, t, k}\right)$. The property of having maximum degree at most $t$ is monotone decreasing; so if we condition on the set $E$ of edges induced by $A \cap B$, then the conditional probability that $A \in \mathcal{S}_{n, t, k}$ is maximized when $E=\emptyset$. Thus,

$$
\mathbb{P}\left(A \in \mathcal{S}_{n, t, k} \mid B \in \mathcal{S}_{n, t, k}\right) \leq \mathbb{P}\left(A \in \mathcal{S}_{n, t, k} \mid E=\emptyset\right) \leq \frac{\mathbb{P}\left(A \in \mathcal{S}_{n, t, k}\right)}{\mathbb{P}(E=\emptyset)}=b^{\left(\begin{array}{l}
\ell \\
2
\end{array}\right)} \mathbb{P}\left(A \in \mathcal{S}_{n, t, k}\right)
$$

(where $b=1 /(1-p)$ ) implying that $p(k, \ell) \leq b^{\left(\begin{array}{l}\ell \\ 2\end{array}\right)}\left(\mathbb{P}\left(A \in \mathcal{S}_{n, t, k}\right)\right)^{2}$. We have though that

$$
\left(\begin{array}{c}
k \\
\ell
\end{array}\right)\left(\begin{array}{l}
n-k \\
k-\ell
\end{array}\right) \leq k^{\ell} \frac{k^{\ell}}{(n-k)^{\ell}}\left(\begin{array}{l}
n \\
k
\end{array}\right)
$$

therefore, it follows that

$$
\Delta_{1} \leq\left(\left(\begin{array}{l}
n \\
k
\end{array}\right) \mathbb{P}\left(A \in \mathcal{S}_{n, t, k}\right)\right)^{2} \sum_{\ell=2}^{\lfloor\lambda \ln n\rfloor}\left(\frac{k^{2}}{n-k}\right)^{\ell} b^{\left(\begin{array}{l}
\ell \\
2
\end{array}\right)}=\left(\mathbb{E}\left(\left|\mathcal{S}_{n, t, k}\right|\right)\right)^{2} \sum_{\ell=2}^{\lfloor\lambda \ln n\rfloor}\left(\frac{k^{2}}{n-k}\right)^{\ell} b^{\left(\begin{array}{l}
\ell \\
2
\end{array}\right) .}
$$

If we set $s_{\ell}=\left(k^{2} /(n-k)\right)^{\ell} b^{\left(\begin{array}{l}\ell \\ 2\end{array}\right)}$, then $s_{\ell+1} / s_{\ell}=b^{\ell} k^{2} /(n-k)$. Thus, the sequence $\left\{s_{\ell}\right\}$ is strictly decreasing for $\ell<\log _{b}(n-k)-2 \log _{b} k$ and is strictly increasing for $\ell>\log _{b}(n-k)-2 \log _{b} k$. So

$$
\max \left\{s_{\ell}: 2 \leq \ell \leq\lfloor\lambda \ln n\rfloor\right\} \leq \max \left\{s_{2}, s_{\lambda \ln n}\right\} .
$$

We have that $s_{2}=b k^{4} /(n-k)^{2}$ and

$$
s_{\lambda \ln n}=\left(\frac{k^{2}}{\sqrt{b}(n-k)} \cdot b^{(\lambda / 2) \ln n}\right)^{\lambda \ln n}=\left(\frac{k^{2} n}{\sqrt{b}(n-k)} \cdot n^{(\lambda / 2) \ln b-1}\right)^{\lambda \ln n} .
$$

Since $k=n^{o(1)}$, it is clear that, if $\lambda<2 / \ln b$, then $s_{\lambda \ln n}=\exp \left(-\Omega\left((\ln n)^{2}\right)\right)$ and, in particular, $s_{\lambda \ln n}=o\left(s_{2}\right)$. Therefore, if $\lambda<2 / \ln b$, then $\Delta_{1}=O\left((\ln n)^{5} / n^{2}\right)\left(\mathbb{E}\left(\left|\mathcal{S}_{n, t, k}\right|\right)\right)^{2}$.

Next, we bound $\Delta_{2}$. We have that

$$
\begin{aligned}
\mathbb{P}\left(A \in \mathcal{S}_{n, t, k} \mid B \in \mathcal{S}_{n, t, k}\right) & \leq \mathbb{P}\left(\forall v \in A \backslash B, \operatorname{deg}_{A}(v) \leq t\right) \leq \mathbb{P}\left(\sum_{v \in A \backslash B} \operatorname{deg}_{A}(v) \leq t(k-\ell)\right) \\
& =\mathbb{P}\left(\operatorname{Bin}(\ell(k-\ell), p)+2 \operatorname{Bin}\left(\left(\begin{array}{c}
k-\ell \\
2
\end{array}\right), p\right) \leq t(k-\ell)\right) .
\end{aligned}
$$

Now we use Lemma 10, with $n_{1}=\ell(k-\ell), n_{2}=\left(\begin{array}{c}k-\ell \\ 2\end{array}\right)$, and $x=t /(k-1)$ which is less than $p$ for $n$ sufficiently large. Note that $n_{1}+2 n_{2}=(k-1)(k-\ell)$ and so $\left(n_{1}+2 n_{2}\right) x=t(k-\ell)$. Hence, by Lemma 10, the last quantity displayed above is at most

$$
\exp \left(-\frac{1}{2}(k-1)(k-\ell) \Lambda^{*}\left(\frac{t}{k-1}\right)\right)=\exp \left(-\left(1-\frac{\ell}{k}\right)\left(\begin{array}{l}
k \\
2
\end{array}\right) \Lambda^{*}\left(\frac{t}{k-1}\right)\right) .
$$


Thus we have shown that

$$
\mathbb{P}\left(A \in \mathcal{S}_{n, t, k} \mid B \in \mathcal{S}_{n, t, k}\right) \leq \exp \left(-\left(1-\frac{\ell}{k}\right)\left(\begin{array}{l}
k \\
2
\end{array}\right) \Lambda^{*}\left(\frac{t}{k-1}\right)\right) .
$$

Also, observe that

$$
\exp \left(\frac{\ell}{k}\left(\begin{array}{l}
k \\
2
\end{array}\right) \Lambda^{*}\left(\frac{t}{k-1}\right)\right)=n^{(1-\delta+o(1)) \ell} .
$$

Therefore, substituting the last two results together with (3) into the expression for $\Delta_{2}$, we obtain

$$
\begin{aligned}
\Delta_{2} & \leq\left(\begin{array}{l}
n \\
k
\end{array}\right)^{2} \mathbb{P}\left(B \in \mathcal{S}_{n, t, k}\right) \exp \left(-\left(\begin{array}{c}
k \\
2
\end{array}\right) \Lambda^{*}\left(\frac{t}{k-1}\right)\right) \sum_{\ell=\lfloor\lambda \ln n\rfloor+1}^{k-1}\left(\frac{k^{2} n^{1-\delta+o(1)}}{n-k}\right)^{\ell} \\
& =\left(\mathbb{E}\left(\left|\mathcal{S}_{n, t, k}\right|\right)\right)^{2} n^{o(\ln n)} \sum_{\ell=\lfloor\lambda \ln n\rfloor+1}^{k-1}\left(n^{-\delta+o(1)}\right)^{\ell}[\text { by Theorem } 7 \text { and } k=O(\ln n)] \\
& \leq\left(\mathbb{E}\left(\left|\mathcal{S}_{n, t, k}\right|\right)\right)^{2} \exp \left(-(\delta \lambda+o(1))(\ln n)^{2}\right)=o\left(1 / n^{2}\right)\left(\mathbb{E}\left(\left|\mathcal{S}_{n, t, k}\right|\right)\right)^{2}
\end{aligned}
$$

for any choice of $\lambda>0$ fixed.

If we let $\lambda=1 / \ln b$, then $\Delta=\Delta_{1}+\Delta_{2}=O\left((\ln n)^{5} / n^{2}\right)\left(\mathbb{E}\left(\left|\mathcal{S}_{n, t, k}\right|\right)\right)^{2}$ and it now follows from (1) and (2) that $\mathbb{P}\left(\alpha^{t}\left(G_{n, p}\right)<k\right) \leq \exp \left(-\Omega\left(n^{2} /(\ln n)^{5}\right)\right)$.

\section{Proof of Theorem 2}

Let $\varepsilon>0$. Note that, by the upper bound of Proposition 1 and the fact that $\Delta\left(G_{n, p}\right) \sim n p$ a.a.s, we have that $\chi^{t}\left(G_{n, p}\right) \leq(1+\varepsilon) n p / t$ a.a.s. This observation trivially gives us the required upper bound of Theorem 2 if $t(n) / \ln n \rightarrow \infty$ as $n \rightarrow \infty$ since then $\kappa_{p}(t / \ln n) \ln n \sim t / p$. The lower bound in this case is implied by the following first moment calculation.

Lemma 11. Fix $0<p<1$ and $\varepsilon>0$. There exists fixed $B=B(p, \varepsilon)$ such that, if $t(n) \geq$ $B \ln n$, then $\chi^{t}\left(G_{n, p}\right) \geq(1-\varepsilon) n p / t$ a.a.s.

Proof. Let $k=k(n)=\lceil t /((1-\varepsilon / 2) p)\rceil+1$ so that $k<t /((1-\varepsilon) p)$ for large enough $n$. Thus, by Lemma $5(\mathrm{i})$, if $\mathcal{S}_{n, t, k}$ is the collection of $t$-dependent $k$-sets in $G_{n, p}$, then

$$
\mathbb{E}\left(\left|\mathcal{S}_{n, t, k}\right|\right) \leq\left(\begin{array}{l}
n \\
k
\end{array}\right) \exp \left(-\left(\begin{array}{l}
k \\
2
\end{array}\right) \Lambda^{*}\left(\frac{t}{k-1}\right)\right) \leq n^{k} \exp \left(-\frac{k^{2}}{3} \Lambda^{*}((1-\varepsilon / 2) p)\right)=\left(n e^{-C t}\right)^{k}
$$

for some fixed $C=C(p, \varepsilon)>0$. Letting $B=2 / C$, we obtain that $\mathbb{E}\left(\mathcal{S}_{n, t, k}\right) \leq n^{-k} \rightarrow 0$ as $n \rightarrow$ $\infty$. So, with probability going to one, $\alpha^{t}\left(G_{n, p}\right) \leq t /((1-\varepsilon) p)$ and $\chi^{t}\left(G_{n, p}\right) \geq(1-\varepsilon) n p / t$.

Therefore, to prove Theorem 2, it suffices to consider $t=t(n)<B \ln n$ where $B=B(p, \varepsilon)$ is as in the above lemma. We may assume that $\varepsilon<1$. Recall that $\kappa_{p}(\tau)$ is continuous on the compact set $[0, B]$ and $\kappa_{p}(\tau) \geq \kappa_{p}(0)>0$ for any $\tau \geq 0$. Hence, there exist $0=\tau_{0}<\tau_{1}<$ $\cdots<\tau_{j}=B$ (for some $j$ ) such that $\kappa_{p}\left(\tau_{i+1}\right) \leq(1+\varepsilon / 3) \kappa_{p}\left(\tau_{i}\right)$ for each $i \in\{0, \ldots, j-1\}$. Since there are only finitely many intervals $\left[\tau_{i}, \tau_{i+1}\right]$, it suffices to consider one, and assume 
that $\tau_{i} \ln n \leq t(n) \leq \tau_{i+1} \ln n$ for all $n$ large enough. By Lemma 8 and the monotonicity of $\chi^{t}$ and $\kappa_{p}(\tau)$,

$$
\begin{aligned}
\chi^{t}\left(G_{n, p}\right) & \leq \chi^{\tau_{i} \ln n}\left(G_{n, p}\right) \leq\left(1+\frac{\varepsilon}{3}\right) \frac{n}{\kappa_{p}\left(\tau_{i}\right) \ln n} \\
& \leq\left(1+\frac{\varepsilon}{3}\right)\left(1+\frac{\varepsilon}{3}\right) \frac{n}{\kappa_{p}\left(\tau_{i+1}\right) \ln n} \leq(1+\varepsilon) \frac{n}{\kappa_{p}(t / \ln n) \ln n}
\end{aligned}
$$

a.a.s. Similarly,

$$
\begin{aligned}
\chi^{t}\left(G_{n, p}\right) & \geq \chi^{\tau_{i+1} \ln n}\left(G_{n, p}\right) \geq\left(1-\frac{\varepsilon}{3}\right) \frac{n}{\kappa_{p}\left(\tau_{i+1}\right) \ln n} \\
& \geq\left(1-\frac{\varepsilon}{3}\right)\left(1+\frac{\varepsilon}{3}\right)^{-1} \frac{n}{\kappa_{p}\left(\tau_{i}\right) \ln n} \geq(1-\varepsilon) \frac{n}{\kappa_{p}(t / \ln n) \ln n}
\end{aligned}
$$

a.a.s. This completes the proof.

\section{Sparse graphs}

In previous sections, we considered dense random graphs, for which the expected average degree is $\Theta(n)$. In this section, we consider random graphs with smaller expected average degree, i.e. $d(n)=n p(n)=o(n)$. For Theorem 3, the upper bound follows from the result of Luczak [18] for the chromatic number, as we already noted. For the lower bound, let us prove more by using the methods of Section 2. First note the following lemma, the elementary proof of which is omitted.

Lemma 12. For any $\tau \geq 0$, there is a unique $\kappa(\tau)>\tau$ such that

$$
\frac{1}{2}\left(\kappa-\tau-\tau \ln \frac{\kappa}{\tau}\right)\left\{\begin{array}{ll}
<1 & \text { if } \tau<\kappa<\kappa(\tau) \\
=1 & \text { if } \kappa=\kappa(\tau) \\
>1 & \text { if } \kappa>\kappa(\tau)
\end{array} .\right.
$$

The function $\kappa(\tau)$ for $\tau \in[0, \infty)$ is continuous and strictly increasing, with $\kappa(0)=2$ and $\kappa(\tau) \sim \tau$ as $\tau \rightarrow \infty$.

Lemma 13. Suppose $0<p(n)<1, p(n)=o(1)$ and let $d(n)=n p(n) \geq 4$ for $n$ sufficiently large. Suppose that $t(n) / \ln d(n) \rightarrow \tau$ as $n \rightarrow \infty$ for some constant $\tau \geq 0$ and let $\kappa$ be a constant such that $\kappa>\kappa(\tau)$ where $\kappa(\tau)$ is as defined in Lemma 12. Then $\chi^{t}\left(G_{n, p}\right) \geq d /(\kappa \ln d)$ a.a.s.

We first show how Theorem 3 follows easily from the above lemma.

Proof of Theorem 3. As noted above, we need only prove the lower bound on $\chi^{t}\left(G_{n, p}\right)$. Since $\kappa(0)=2$ and $\kappa(\cdot)$ is continuous and strictly increasing, we may choose $\tau>0$ so that $2<$ $\kappa(\tau)<2 /(1-\varepsilon)$. By Lemma 13, there exists a constant $d_{0}$ such that, if $d(n) \geq d_{0}$, then $\chi^{\tau \ln d}\left(G_{n, p}\right) \geq d /((2 /(1-\varepsilon)) \ln d)$. The theorem now follows from the monotonicity of $\chi^{t}$. 
Proof of Lemma 13. Let $k=k(n)=\lceil\kappa \ln d(n) / p\rceil+1$ where $\kappa>\kappa(\tau)$. Since $\kappa>\tau$, it follows that $t(n) \leq p(n)(k(n)-1)$ for large enough $n$. By the Chernoff Inequality (2.6) of [13],

$$
\begin{aligned}
\mathbb{P}\left(\overline{\operatorname{deg}}\left(G_{k, p}\right) \leq t\right) & \leq \exp \left(-\left(\begin{array}{l}
k \\
2
\end{array}\right) p\left(\frac{t}{p(k-1)} \ln \frac{t}{p(k-1)}+1-\frac{t}{p(k-1)}\right)\right) \\
& =\exp \left(-\left(\frac{\kappa}{2}\left(\frac{\tau}{\kappa} \log \frac{\tau}{\kappa}+1-\frac{\tau}{\kappa}\right)+o(1)\right) k \ln d\right) .
\end{aligned}
$$

(To apply the inequality from [13], we put $\lambda=\left(\begin{array}{c}k \\ 2\end{array}\right) p$ and $t / \lambda=1-t(n) /(p(k-1)$ ), where $t(n)$ is 'our' $t$.) Let $\delta=(\kappa-\tau-\tau \ln (\kappa / \tau)) / 2-1$, so that $\delta>0$ by Lemma 12 . We have just seen that $\mathbb{P}\left(\overline{\operatorname{deg}}\left(G_{k, p}\right) \leq t\right) \leq \exp (-(\delta+1+o(1)) k \ln d)$. Now, en $/(k d)<e /(\kappa \ln d)<1$ since $\kappa \geq 2$ and $d \geq 4$; and so $\ln \left(\begin{array}{l}n \\ k\end{array}\right) \leq k \ln (e n / k) \leq k \ln d$. It follows that, if $\mathcal{S}_{n, t, k}$ is the collection of $t$-dependent $k$-sets in $G_{n, p}$, then $\ln \mathbb{E}\left(\left|\mathcal{S}_{n, t, k}\right|\right) \leq(-\delta+o(1)) k \ln d \rightarrow-\infty$ as $n \rightarrow \infty$ and this completes the proof.

\section{Concluding remarks}

In this paper, we have provided a detailed description of the behaviour of the $t$-improper chromatic number of dense random graphs $G_{n, p}$ over the range of choices for the growth of $t=t(n)$. We also briefly considered the $t$-improper chromatic number of sparse random graphs. In this setting, perhaps the lower bound implied by Lemma 13 is tight? This would correspond to a sparse analogue of Lemma 8. In Section 4.3 of [14], we showed that a sparse analogue of Theorem 7 holds as long as $p=n^{-o(1)}$ (cf. Theorem 4.17). It is straightforward to adapt the proof of Lemma 8 to this range of $p$, but it is an open problem to extend the result to smaller choices of $p$.

Our results contrast with the behaviour of random geometric graphs, where $\chi^{t}$ is likely to be close to $\chi /(t+1)$ for $t$ smaller than the expected average degree - see [16].

\section{Acknowledgement}

We would like to thank the referee for a careful reading and helpful comments.

\section{References}

[1] J. A. Andrews and M. S. Jacobson. On a generalization of chromatic number. In Proceedings of the Sixteenth Southeastern International Conference on Combinatorics, Graph Theory and Computing (Boca Raton, Fla., 1985), Congr. Numer., volume 47, pages 33-48, 1985.

[2] B. Bollobás. The chromatic number of random graphs. Combinatorica, 8(1):49-55, 1988.

[3] B. Bollobás. Random Graphs, volume 73 of Cambridge Studies in Advanced Mathematics. Cambridge University Press, Cambridge, 2nd edition, 2001.

[4] B. Bollobás and A. Thomason. Generalized chromatic numbers of random graphs. Random Structures Algorithms, 6(2-3):353-356, 1995.

[5] L. Cowen, W. Goddard, and C. E. Jesurum. Defective coloring revisited. J. Graph Theory, 24(3):205-219, 1997. 
[6] L. J. Cowen, R. H. Cowen, and D. R. Woodall. Defective colorings of graphs in surfaces: partitions into subgraphs of bounded valency. J. Graph Theory, 10(2):187-195, 1986.

[7] A. Dembo and O. Zeitouni. Large Deviations Techniques and Applications, volume 38 of Applications of Mathematics (New York). Springer-Verlag, New York, 2nd edition, 1998.

[8] N. Eaton and T. Hull. Defective list colorings of planar graphs. Bull. Inst. Combin. Appl., 25:79-87, 1999.

[9] N. Fountoulakis, R. J. Kang, and C. McDiarmid. The $t$-stability number of a random graph. Available at http://arxiv.org/abs/0809.0141. Submitted, August 2008.

[10] G. R. Grimmett and C. J. H. McDiarmid. On colouring random graphs. Math. Proc. Cambridge Philos. Soc., 77:313-324, 1975.

[11] F. Harary. Conditional colorability in graphs. In Graphs and Applications (Boulder, Colo., 1982), Wiley-Intersci. Publ., pages 127-136. Wiley, New York, 1985.

[12] F. Harary and K. F. Jones. Conditional colorability. II. Bipartite variations. In Proceedings of the Sundance Conference on Combinatorics and Related Topics (Sundance, Utah, 1985), Congr. Numer., volume 50, pages 205-218, 1985.

[13] S. Janson, T. Łuczak, and A. Rucinski. Random Graphs. Wiley-Interscience Series in Discrete Mathematics and Optimization. Wiley-Interscience, New York, 2000.

[14] R. J. Kang. Improper Colourings of Graphs. PhD thesis, University of Oxford, 2008. http://ora.ouls.ox.ac.uk/objects/uuid:a93d8303-0eeb-4d01-9b77-364113b81a63.

[15] R. J. Kang and C. J. H. McDiarmid. The $t$-improper chromatic number of random graphs. In Proceedings of the 4 th European Conference on Combinatorics, Graph Theory and Applications (Seville, 2007), Electron. Notes Discrete Math., volume 29, pages 419425, 2007.

[16] R. J. Kang, T. Müller, and J.-S. Sereni. Improper colouring of (random) unit disk graphs. Discrete Math., 308(8):1438-1454, 2008.

[17] L. Lovász. On decompositions of graphs. Studia Sci. Math. Hungar., 1:237-238, 1966.

[18] T. Euczak. The chromatic number of random graphs. Combinatorica, 11(1):45-54, 1991.

[19] D. Matula and L. Kučera. An expose-and-merge algorithm and the chromatic number of a random graph. In Random Graphs '87 (Poznań, 1987), pages 175-187. Wiley, Chichester, 1990.

[20] C. McDiarmid. Concentration. In Probabilistic methods for algorithmic discrete mathematics, volume 16 of Algorithms Combin., pages 195-248. Springer, Berlin, 1998.

[21] E. R. Scheinerman. Generalized chromatic numbers of random graphs. SIAM J. Discrete Math., 5(1):74-80, 1992.

[22] R. Škrekovski. List improper colourings of planar graphs. Combin. Probab. Comput., 8(3):293-299, 1999. 


\section{A Proofs of two large deviations tools}

Proof of Lemma 4. We first prove the right inequality. The following basic inequality is well known (cf. (2.4) of [13] or Lemma 2.2 of [20]). Let $X \in \operatorname{Bin}(n, p)$, where $0<p<1$. Then for $0 \leq t<q$

$$
\mathbb{P}(X \geq n p+n t) \leq\left(\left(\frac{p}{p+t}\right)^{p+t}\left(\frac{q}{q-t}\right)^{q-t}\right)^{n} .
$$

It follows that for $0 \leq t<p$

$$
\begin{aligned}
\mathbb{P}(X \leq n p-n t) & =\mathbb{P}(n-X \geq n q+n t)=\mathbb{P}(Y \geq n q+n t) \\
& \leq\left(\left(\frac{q}{q+t}\right)^{q+t}\left(\frac{p}{p-t}\right)^{p-t}\right)^{n}=\exp \left(-n \Lambda^{*}(p-t)\right),
\end{aligned}
$$

where $X \in \operatorname{Bin}(n, p)$ and $Y \in \operatorname{Bin}(n, q)$. Thus, putting $t=p-k / n$ we obtain

$$
\mathbb{P}(X \leq k)=\mathbb{P}(X \leq n p-n t) \leq \exp \left(-n \Lambda^{*}(k / n)\right) .
$$

For the left inequality, we use the sharp form of Stirling's formula due to Robbins, see for example inequality (1.4) of [3]. Note that $1 /(12 k)+1 /(12(n-k))=n /(12 k(n-k))$; thus, for $1 \leq k \leq n-1$,

$$
\begin{aligned}
\left(\begin{array}{l}
n \\
k
\end{array}\right) p^{k} q^{n-k} & \geq\left(\frac{n p}{k}\right)^{k}\left(\frac{n q}{n-k}\right)^{n-k}\left(\frac{n}{2 \pi k(n-k)}\right)^{1 / 2} \exp \left(-\frac{n}{12 k(n-k)}\right) \\
& \geq \exp \left(-n \Lambda^{*}(k / n)\right)(2 \pi)^{-1 / 2} \max \left\{k^{-1 / 2},(n-k)^{-1 / 2}\right\} e^{-1 / 6} \\
& \geq \delta \cdot \max \left\{k^{-1 / 2},(n-k)^{-1 / 2}\right\} \cdot \exp \left(-n \Lambda^{*}(k / n)\right)
\end{aligned}
$$

where $\delta=(2 \pi)^{-1 / 2} e^{-1 / 6}$.

Proof of Lemma 10. Let $\bar{X}=n_{1}-X$ so $\bar{X} \in \operatorname{Bin}\left(n_{1}, q\right)$, and let $\bar{Y}=2 n_{2}-Y$ so $\bar{Y} / 2 \in$ $\operatorname{Bin}\left(n_{2}, q\right)$. Then $\mathbb{P}\left(X+Y \leq\left(n_{1}+2 n_{2}\right) x\right)=\mathbb{P}\left(\bar{X}+\bar{Y} \geq\left(n_{1}+2 n_{2}\right)(1-x)\right)$. Now for any real $u$ we have

$$
\left(p+q e^{u}\right)^{2}=p+q e^{2 u}-p q\left(1-e^{u}\right)^{2} \leq p+q e^{2 u},
$$

and so

$$
\mathbb{E}\left(e^{u \bar{X}}\right)=\left(p+q e^{u}\right)^{n_{1}} \leq\left(p+q e^{2 u}\right)^{n_{1} / 2} .
$$

Thus, $\mathbb{E}\left(e^{u(\bar{X}+\bar{Y})}\right) \leq\left(p+q e^{2 u}\right)^{\left(n_{1}+2 n_{2}\right) / 2}$. Hence, for any $u \geq 0$, by Markov's inequality,

$$
\begin{aligned}
\mathbb{P}\left(\bar{X}+\bar{Y} \geq\left(n_{1}+2 n_{2}\right)(1-x)\right) & \leq e^{-u\left(n_{1}+2 n_{2}\right)(1-x)}\left(p+q e^{2 u}\right)^{\left(n_{1}+2 n_{2}\right) / 2} \\
& =\left(e^{-2 u(1-x)}\left(p+q e^{2 u}\right)\right)^{\left(n_{1}+2 n_{2}\right) / 2}
\end{aligned}
$$

Note that $p(1-x) /(q x) \geq 1$. Now choose $u \geq 0$ such that $e^{2 u}=p(1-x) /(q x)$ to obtain the desired inequality. 